\title{
Computerized Waste Segregation Robot
}

\author{
Saurabh Wasnik ${ }^{1}$, Dushyantsingh Thakur ${ }^{2}$, Prof. Swapnil V. Deshmukh ${ }^{3}$ \\ Department of Computer Science \& Engineering, SBG University, Amravati, India ${ }^{1,2,3}$
}

\begin{abstract}
The major dilemmas faced due to improper waste management include health risks to humankind and environmental concerns. We in India generates over 150,000 tonnes of municipal solid waste per day, with Mumbai being the world's fifth most wasteful city. Yet, only $83 \%$ of the waste is collected and less than $30 \%$ is treated. According to the World Bank, India's daily waste generation will reach 377,000 tonnes by 2025 . Now blame urbanization and industrialization, but the consequences of India's megacities producing tonnes of waste are tangible and troubling. The unprocessed waste contains different types of solid waste like metallic \& organic material, which can be used for recycling and composting purpose. As it's very necessary to segregate the solid waste with a more efficient and intelligent approach. The robots are very much capable of doing such things on behalf of the human. Robots are easily programmable to do such tasks. By using the robots, the reduction of manpower will take place with the prevention of human contacts with chemically hazardous substances. Robots use technologies like artificial intelligence \& machine learning to achieve such a complex task. These technologies empower machines a smart understanding to think like a human being. By developing such understanding for a robot, we can assume that in the future a robot can take place of the human in such a place of work where humans suffer most physically and mentally.
\end{abstract}

Keywords: Computerized, Machine learning, Solid Waste, Robotic Arm, Segregation, Microcontroller.

\section{INTRODUCTION}

Technology like Machine learning has given us an exceptional opportunity to bring such things in existence which can improve our lives. The proposed system is the true aspect of solving problems by today's technologies in which a robot is able to perform a hectic and complex task on behalf of a human. Using robots for such task brings more efficiency and accuracy in the work. Though the separation of waste using a robot is kind of an obvious future which will lead our society towards the increase in the health and improvisation of our environment. Machine learning and robotic both sections of technologies have magnificent features \& advantages when combining together.

In our proposed system we have to build a robotic arm which is able to detect and separate the waste in biodegradable and non- biodegradable form. The separated solid waste classified as non-biodegradable like plastic, metallic cans etc. can be used for recycling purpose whereas other waste which is been identified as biodegradable can be used for composting purpose and for some other. The existing system has huge machinery which occupies a large amount of space and makes more sound. This system needs $24 \mathrm{hr}$ of monitoring and high maintenance on a regular basis. The complete system setup needs too much costing and manpower for operating. Operating such systems requires lots of skilled people. Even after deploying such a huge amount of resources we are not getting satisfactory output.

\section{PROPOSED SYSTEM}

The proposed system has a programmable robotic arm which is able to detect the object (waste) and separate it. The system has a rounded rotating platform which stops automatically for scanning the object when an object comes above the magnetic sensor. As the platform stop the webcam scan the object and separate it with the help of a robotic arm. The system is able to classify the object with an image processing technique which is implemented by using OpenCV with python. The OpenCV is a strong \& reliable tool for such a system. Our proposed system is able to separate the waste into biodegradable and non-biodegradable waste. All the activates is been controlled and managed by Arduino Uno. The micro-controller is the heart of the system which is powerful and easy to program. It is embedded on the motherboard and all other pieces of equipment are connected to the motherboard with the help of wires. Bins are used for collecting the waste. 
Vol. 8, Issue 4, April 2019

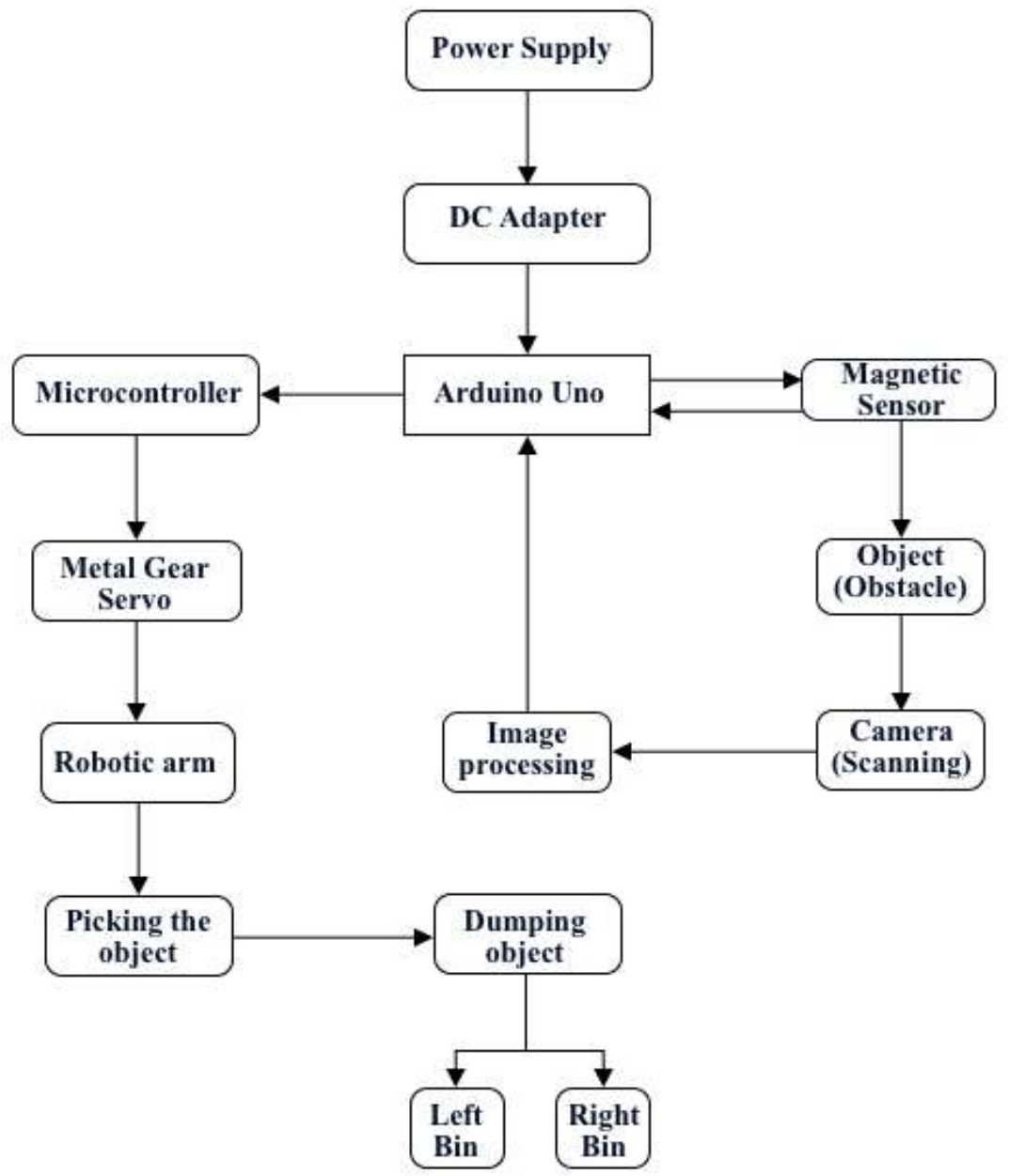

Fig. 1.1 System Flow Chart

A. System Requirements Analysis

The computerized waste segregation robot can be categorized in a mobile robot which tackles some specific kind of object with the help of image processing technique. The listed following things are the required parts for building the robot for assembling of the robot.

- 1 Arduino Uno kit

- 2 High Torque Gear Ball Bearing Servo

- 1 Dc Motor for rotating Platform

- 1 metallic Circular Robotic gripper

- 2 Micro Servo $17 \mathrm{~g}$ Servo for wrist rotation

- 1 Transistor with power cable

- 1 DC Adapter

- 1 Hall Magnetic Sensor

- 1 Webcam

- Laptop with Strong GPU \& Linux OS

B. Hardware \& Software Analysis

- The Arduino UNO board with ATmega328 Micro-controller [1] has a major advantage of 14 input/output of which 6 can be used as PWM outputs pins and of which 6 can be used as PWM outputs [2].

- The robotic arm and a circular metallic gripper are suitable to pick the small and medium size of waste objects [5].

- And the Image processing technique is achieved by using OpenCV tool with python which helps to classify the waste into biodegradable and non-biodegradable [3].

- Implementing the image processing in Linux OS offers more functionality and easiness to execute the core methodology as per us convince. Along with this it also performs well with embedded system as we are using a microcontroller. 


\section{International Journal of Advanced Research in Computer and Communication Engineering}

Vol. 8, Issue 4, April 2019

\section{Robotic Arm}

The robotic arm is been develop with the help 4 servo motors in which 1 is for turning the left and right in bottom, 1 is for elbow for up and down motion and finally two for gripper which will operate both parts of the gripper. All motors are connected to Arduino Uno board which control all the system activates. The motors we are using called MG996R which is an upgraded version of the famous MG995 and features which are upgraded as shock-proofing and redesigned PCB and IC control system that makes it much more accurate and precise.



Fig. 1.2 MG996R Moto Servo

\section{CORE TECHNOLOGIES}

Image processing with OpenCV is the best approach for segregation of solid waste in specific forms. The segregation we are looking for is biodegradable and non-biodegradable. But solid waste that we are going to process consist of different types of contents. So, we use some advanced technologies like machine learning and artificial intelligence.

\section{A. Image Processing}

Image processing technique helps us to identify the objects according to our desired output. In our proposed system image processing is implemented by OpenCV with python programming which offers more stability and functionality to our system as python has rich datasets and libraries for performing such tasks. Mainly image processing consists of scanning and analysing the object.

\section{B. OpenCV}

Computer Vision is the science of programming the computer to process and understand the images just by training. OpenCV offers us such opportunity for creating exciting new possibilities in technology. An OpenCV has wide and very useful features which can process the images very accurately and deliver the accurate result for proper identification of object even in real time. The OpenCV goes very well when it is implemented using a Python programming language which is also known as a future programming language. The reason behind using python is it has a rich set of libraries such as panda, NumPy etc. This various library helps us to improve the efficiency of image processing by extracting a different kind of features of image [6].

\section{Machine Learning in Image Processing}

The support vector machines (SVMs) is, among others, very popular and conceptually machine learning tool for image processing. The support vector machines based on finding a set of hyperplanes to separate the sets of positive and negative examples. Today's image processing tradition involving machine learning for real-time performance almost exclusively uses AdaBoost instead of SVMs. Though SVM is easier to program and has proven itself to work well. Which make SVM used in most of research paper regarding to image processing. This makes the SVMs approach a better choice for real-time applications. A number of recent papers, using both AdaBoost and SVMs, confirm the same, and even apply a two-phase process. Most windows are processed in the first phase by AdaBoost, and in the second phase, an SVM is used on difficult cases that could not be easily eliminated by AdaBoost. This way, the realtime constraint remains intact [7]. 


\section{International Journal of Advanced Research in Computer and Communication Engineering}

Vol. 8, Issue 4, April 2019

\section{IMAGE FEATURE EXTRACTION}

Image feature extraction is very much important for identifying the object in details for better scanning and segregation. Features are going to be extracted by image processing technique using OpenCV programming.

\section{A. Colour feature extraction}

$\mathrm{R}, \mathrm{G}, \mathrm{B}$ projection values are been extracted by OpenCV programming and stored in the database. For better accuracy, we need to gather a maximum sample of images. So that the system will have a large amount of knowledge of identifying the image category.

\section{B. Size feature extraction}

Size feature extraction helps to analyse the size of objects such as vegetables, fruits, and many other contents. We can get the same object at a various size so that we need to train the system at various sizes of object this will help system to be more precise while segregating the various size of same and different object.

\section{Texture feature extraction}

The texture feature extraction is based on multichannel filtering, which copies some aspects of the human optical system. The human optical system crumbles an image created in the retina into several refined images, each of them having alterations in depth within a limited range of frequencies and orientation.

\section{WORKING PRINCIPLE}

The system awakes as the commands receive which start rotating the platform and bring the solid waste in front of the webcam. As the solid waste come under the webcam fitted above it. It starts scanning the solid waste. It compares the image with the trained dataset. As we have already stored some images of solid waste the system compares the new images to the images taken before and with the of image processing, the analysis of current object starts which perform operation internally like features extraction in which the image is processed for obtaining the features like colour, size, and texture. On the basis of this features the object is classified and the decision is made whether the scanned waste is biodegradable or non-biodegradable. As the decision is made by the system the robot receives the command of picking up the object and separates it from the platform and dump waste in the specific bin.

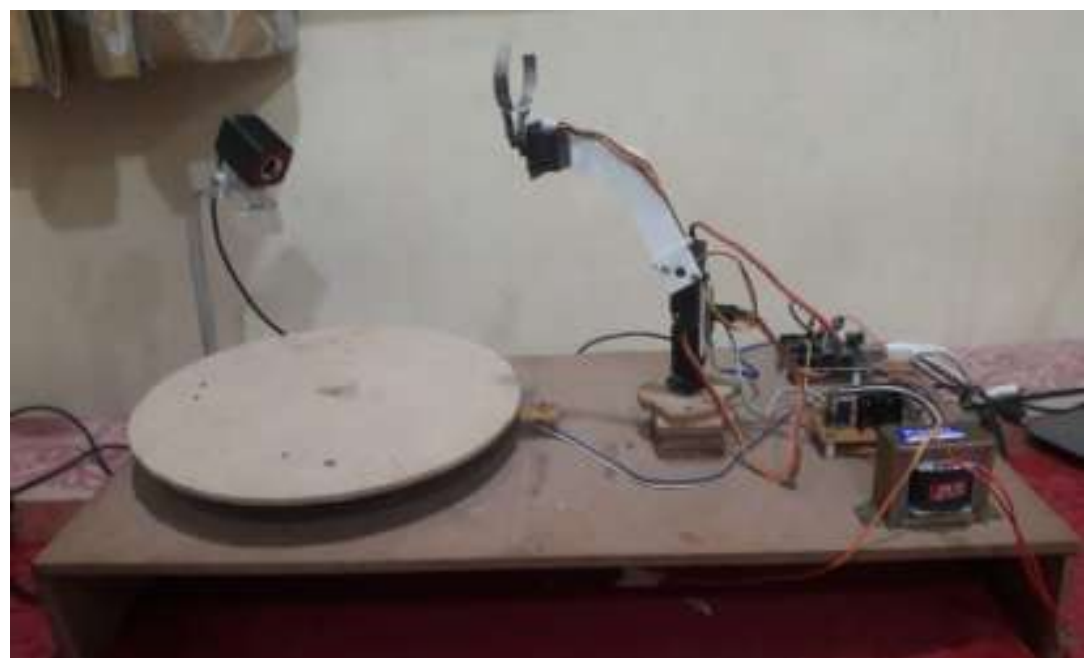

Fig. 1.3 Computerised Waste Segregation Robot

\section{CONCLUSIONS}

Finally, we can say that robotics is the future of waste management. And this will definitely bring some difference in our environment and civilization. System like this will also reduce the human efforts and bring more efficiency and accuracy in waste segregation. So, developing such robotic system with todays advanced technologies we can definitely bring the huge and drastic change is waste management which is very major problem in today's world specially in India. This will also produce the opportunities of employment in municipal waste management sector with smart and safe approach. And at last our moto behind developing such system is to inspire the young generation to solve such social issues with emerging technologies \& also understands the importance of their nature. 


\section{ACKNOWLEDGMENT}

The authors are grateful to the body of management and department of computer science \& engineering of Prof. Ram Meghe Institute of Technology \& Research, Badnera-Amravati. For guiding and supporting us in very possible manner. We would also be like to thanks our honourable HoD Dr. G. R. Bamnote for giving me the opportunity to embark upon this topic and for his continued encouragement throughout the preparation of project.

\section{REFERENCES}

[1]. B.P. Singh, Microprocessors and Microcontrollers, Galcotia Publications (P) Ltd, First edition, New Delhi, 1997.

[2]. Embedded Controller Hand book, Intel Corporation, USA.

[3]. Saravana kannan G, Sasi kumar S, Ragavan R, Balakrishnan M “Automatic Garbage Separation Robot Using Image Processing Technique" International Journal of Scientific and Research Publications, Volume 6, Issue 4, ISSN 2250-3153, April 2016.

[4]. Gareth J.Monkman, Stefan Hesse, Ralf Steinmann, Henrikschunk "Robot Grippers” ISBN:978-3-527-40619-7,pp 463 November 2006

[5]. G.K. Dubey "Fundamental Electrical Drives" second edition 2002, Narosa Publications, Second edition, 2002.

[6]. R. Laganière. OpenCV 2 Computer Vision Application Programming Cookbook. Packt Publishing 2011.

[7]. R. Szeliski. Computer Vision: Algorithms and Applications. Springer 2011. 